\title{
Growth promotion of common bean and genetic diversity of bacteria from
}

\section{Amazon pastureland}

\author{
Linnajara de Vasconcelos Martins Ferreira ${ }^{1}$, Fernanda de Carvalho², Júlia Fonseca Colombo Andrade², Fatima Maria de Souza Moreira²*
}

${ }^{1}$ Federal Institute of Pará - Campus Marabá Rural, BR 155 km 25, PA 26 de Março, C.P. 41 - 68508-979 - Marabá, PA - Brazil.

${ }^{2}$ Federal University of Lavras - Dept. of Soil Science - Sector of Biology, Microbiology and Biological Processes, C.P. 3037 - 37200-000 - Lavras, MG - Brazil.

*Corresponding author <fmoreira@dcs.ufla.br>

Edited by: Fernando Dini Andreote

Received February 07, 2017

Accepted July 24, 2017

\begin{abstract}
A significant number of bacterial species, particularly in the rhizosphere, may benefit plant growth and development. This group of bacteria is known as plant growth-promoting rhizobacteria (PGPR). This study identified genetically isolates of common bean nodules used to trap bacteria from Amazon pastureland and investigated their capacity of nodulating and promoting growth of common bean when inoculated or co-inoculated with CIAT899 strain (Rhizobium tropici). Two experiments were carried out in a greenhouse, in axenic conditions, using the common bean cultivar Talismã. In the first experiment, 56 PGPR strains were evaluated individually regarding growth promotion and nodulation. In the second experiment, inoculation of seven PGPR strains previously selected in the first experiment was evaluated in three forms of $\mathrm{N}$ supply: Co-inoculation with CIAT 899 in the presence of low N-mineral concentration; individual inoculation in the presence of high $\mathrm{N}$-mineral concentration; and individual inoculation in the presence of low N-mineral concentration. The 16S rRNA gene sequencing showed predominance of Pseudomonas genus, identified in $35 \%$ of the sequenced strains. Other genera were identified: Rhizobium, Burkholderia, Xanthomonas and Bacillus. Inoculation of the seven strains with CIAT 899 promoted distinct plant growth in different forms of $\mathrm{N}$ supply. In addition, $\mathrm{N}$-mineral supply can be replaced by co-inoculation with strains of Pseudomonas sp. (UFLA 02-281 and UFLA 02293) and Bacillus sp. (UFLA 02-298) identified in this study.
\end{abstract}

Keywords: Phaseolus vulgaris, co-inoculation, endophytic bacteria, rhizobia

\section{Introduction}

In the search for promising isolates that promote plant growth, the Amazon region stands out for its high diversity of organisms in the soil, including microorganisms. This fact was observed in the diversity of rhizobia genera and strains found in western Amazon (Guimarães et al., 2012; Jaramillo et al., 2013). Besides, in the Amazon region, expressive area of the soil is used for pastureland, where recent studies have found a high bacterial diversity (Carvalho et al., 2016; Soares et al., 2016).

Studies show that bacterial strains isolated from this region have the potential to act as plant growth promoters in the biological nitrogen fixing (BNF) process (Ferreira et al., 2012) or in other processes, such as inhibition of phytopathogenic fungal growth, solubilization of phosphates (Marra et al., 2012), production of indole3-acetic acid (IAA) (Silva et al., 2012; Oliveira-Longatti et al., 2013) and having adaptation to abiotic stresses (Medeiros et al., 2011; Soares et al., 2014).

Co-inoculation of Rhizobium and plant growth-promoting rhizobacteria (PGPR) in legumes have received more attention in recent years (Tilak et al., 2006; Mishra et al., 2011; Samavat et al., 2012). This combination brings positive effects on the cultivation of legume species, since it provides growth and nutrient absorption, as it happens in lentil plants with the co-inoculation of Pseudomonas sp. with Rhizobium leguminosarum (Mishra et al., 2011), increases of nodulation in some crops, such as in pigeon pea [(Cajanus cajan (L.) Mill sp.)], with the co-inoculation of Pseudomonas fluorescens with Rhizobium sp. (Tilak et al., 2006). Besides, this combination presents positive effects on grain yield, as it happens in bean crop, with the co-inoculation of Pseudomonas and Rhizobium (Samavat et al., 2012).

In Brazil, three Rhizobium strains are authorized as inoculants for the common bean: CIAT 899, PRF 81, and $\mathrm{H}$ 12. Results show that bean plants can benefit from $\mathrm{BNF}$ in the field, with no need of $\mathrm{N}$ fertilizers application. The challenge is to apply appropriate management of this symbiosis to increase its efficiency in $\mathrm{N}$ supply to plants. Co-inoculation of Rhizobium and PGPR may be a strategy to improve efficiency of these strains.

The objective of this study was to genetically identify isolates from nodules of common bean inoculated with Amazon pasture land soil, and to verify their ability to nodulate and promote growth of bean plants coinoculated or not with CIAT 899.

\section{Materials and Methods}

\section{Strains origin}

The strains used in this study (56) were isolated from inside the nodules of the common bean inoculated with soils from the Amazon region under pasture systems (Moreira et al., 2009).

\section{S rRNA gene sequencing of bacterial strains}

Bacterial strains were grown in medium 79 (Fred and Waksman, 1928). DNA of 56 bacterial strains was extracted by the alkaline lysis method (Niemann et al., 
1997). The $16 \mathrm{~S}$ rRNA gene was partially amplified with final reaction volume of $50 \mu \mathrm{L}$. The following concentrations were: $5 \mu \mathrm{L}$ DNA, $5 \mu \mathrm{L} 10 \mathrm{X}$ buffer for each PCR, $5 \mu \mathrm{L}$ dNTP Mix (0.2 mM of each dNTP), $5 \mu \mathrm{L} \mathrm{MgCl}_{2}(2.5$ $\mathrm{mM}), 1 \mu \mathrm{L}$ of each primer $\left(10 \mathrm{mmol} \mathrm{L}^{-1}\right)(27 \mathrm{~F}$ primer (5'-AGAGTTTGATCCTGGCTCAG-3') and 1492R primer (5'-GGTTACCTTGTTACGACTT-3') (Lane, 1991), $0.4 \mu \mathrm{L}$

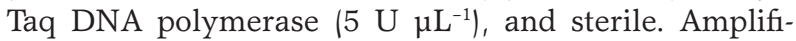
cation reaction occurred following initial denaturation (94 ${ }^{\circ} \mathrm{C}$ for $\left.5 \mathrm{~min}\right), 40$ denaturation cycles $\left(94^{\circ} \mathrm{C}\right.$ for $40 \mathrm{~s}$ ), annealing $\left(55^{\circ} \mathrm{C}\right.$ for $\left.40 \mathrm{~s}\right)$, extension $\left(72^{\circ} \mathrm{C}\right.$ for $\left.1.5 \mathrm{~min}\right)$ and final extension $\left(72^{\circ} \mathrm{C}\right.$ for $\left.7 \mathrm{~min}\right)$. Reaction was performed in thermocycler. PCR products were separated in $1 \%$ agarose gel and visualized under UV light.

PCR products were sequenced using the oligonucleotide primer $27 \mathrm{~F}$ and $1492 \mathrm{R}$. Sequence quality was evaluated using the BioNumerics software (version 7.1). Additionally, the sequences were subjected to BLAST to compare with similar sequences deposited in the GenBank database (National Center for Biotechnology Information - NCBI) (http://www.ncbi.nlm.nih.gov) and were deposited under the accession numbers KU613374 to KU613408.

\section{Experiment 1: Authentication and promotion of vegetative growth of common bean cultivar Talismã plants: in vitro assay}

The experiment was carried out in a greenhouse from June to July 2014 and was conducted for 40 days. Plants were cultivated in sterilized $500 \mathrm{~mL}$ long neck bottles. The experimental design was completely randomized, with three replications and 59 treatments. Treatments consisted of inoculations with 56 bacterial strains isolated from bean nodules (Table 1); one reference strain approved by MAPA (Brazilian Ministry of Livestock Agriculture and Supply) as inoculum for bean crops; and two negative controls without inoculation, with high and low N-mineral concentrations. The experiment used the following stock solutions of $\mathrm{N}$-mineral sources: $\mathrm{Ca}\left(\mathrm{NO}_{3}\right)_{2} \cdot 4 \mathrm{H}_{2} \mathrm{O}$ mol L-1 : $236.6 \mathrm{~g} \mathrm{~L}^{-1} ; \mathrm{KNO}_{3} \mathrm{~mol} \mathrm{~L}^{-1}: 101.111 \mathrm{~g} \mathrm{~L}^{-1}$ and $\mathrm{NH}_{4} \mathrm{H}_{2} \mathrm{PO}_{4}$ mol L-1: $132.14 \mathrm{~g} \mathrm{~L}^{-1}$. From these stock solutions, high $\mathrm{N}$ concentration solution received respectively: $4 ; 0.6$ and $0.1 \mathrm{~mL} \mathrm{~L}^{-1}$, while low $\mathrm{N}$ concentration solution received: $0.1 ; 0.125$ and $0.25 \mathrm{~mL} \mathrm{~L}^{-1}$.

In the inoculated treatments, and in the control without inoculation and with low $\mathrm{N}$ concentration, Hoagland (Hoagland and Arnon, 1950) nutrient solution with low $\mathrm{N}$ concentration (5.25 $\mathrm{mg} \mathrm{L}^{-1}$ ) was applied. In the control with high $\mathrm{N}$-mineral concentrations, complete Hoagland solution was used, with $52.5 \mathrm{mg} \mathrm{L}^{-1} \mathrm{ni}-$ trogen. Subsequently, all bottles were autoclaved for 60 min at a pressure of $1.5 \mathrm{~kg} \mathrm{~cm}^{2}$, at $121^{\circ} \mathrm{C}$.

Long neck bottles containing $500 \mathrm{~mL}$ Hoagland nutrient solution were covered with aluminum foil, and two filter paper strips $(2 \mathrm{~cm}$ in width and length equal to the bottle height) were put inside each bottle to support roots and promote contact of the plant with the nutrient solution. Before sowing, bean seeds (cv. Talismã) were surface disinfected using $98 \%$ ethanol (30 s), $2 \%$ sodium hypochlorite ( $2 \mathrm{~min}$ ) and then subjected to successive rinses in autoclaved distilled water (Guimarães et al., 2012). After disinfection, seeds were placed in sterile Petri dishes containing moistened filter paper and cotton, where they remained for $48 \mathrm{~h}$ in a growth chamber at $28{ }^{\circ} \mathrm{C}$, for rootlets emergence.

For inoculum preparation, bacterial strains were cultivated in liquid culture medium 79 (Fred and Waksman, 1928), stirred at $110 \mathrm{rpm}$, at $28{ }^{\circ} \mathrm{C}$, for 3 days. At sowing, $1 \mathrm{~mL}$ of the inoculant $\left(10^{9}\right.$ Cells $\left.\mathrm{mL}^{-1}\right)$ was added to the pre-germinated seeds in the inoculated treatments. In the controls without inoculation, $1 \mathrm{~mL}$ of the culture medium without inoculum was applied.

Thirty-five days (pre-flowering stage) after the experiment beginning, plants were harvested to determine the following traits: number of nodules $(\mathrm{NN})$; nodule dry matter (NDM); shoot dry matter (SDM); root dry matter (RDM); and total dry matter (TDM). To determine NN, nodules were detached from roots and counted. To determine NDM, SDM and RDM, nodules, shoot and roots were placed in paper bags and allowed to dry in forced air circulation oven at $60^{\circ} \mathrm{C}$ until constant weight. Data were subjected to analysis of variance, using the statistical software SISVAR version 5.1 (Ferreira, 2011). Treatments were compared by the Scott-Knott test at $5 \%$ probability. NN and NDM values had been previously transformed into square root of $(\mathrm{Y}+0.5)$.

\section{Experiment 2: Inoculation and co-inoculation of bean with plant growth-promoting bacteria}

To evaluate plant growth promotion potential subjected to inoculation and co-inoculation with bean inoculant rhizobia strain (CIAT 899), seven strains were selected (UFLA 02-274, UFLA 02-276, UFLA 02-281, UFLA 02-282, UFLA 02-298, UFLA 02-290, UFLA 02293) from those that stood out for shoot and root dry matter, as observed in experiment 1 , as well as, from those that had already been identified by sequencing, at the time the experiment was carried out.

The experiment was carried out in Oct and Nov, 2014, in autoclaved Leonard jars (Vincent, 1970), containing $700 \mathrm{~mL}$ Hoagland solution (Hoagland and Arnon, 1950 ) in the bottom and a mixture of sand and vermiculite $(1: 2)$ in the upper part. The experiment was conducted for 50 days. The experimental design was completely randomized in a factorial design $(8 \times 3)$ with three replications. Factor 1 was the individual inoculation of seven strains, plus one treatment without inoculation. Factor 2 was three forms of $\mathrm{N}$ supply: low $\left(5.25 \mathrm{mg} \mathrm{L}^{-1}\right)$ and high (52.5 $\left.\mathrm{mg} \mathrm{L}^{-1}\right) \mathrm{N}$-mineral concentration in the nutrient solution and co-inoculation with CIAT899 with low $\mathrm{N}$-mineral concentration.

Bean seeds (cv. Talismã) were surface disinfected as described in experiment 1 . In each jar, four seeds were sown and inoculated with $1 \mathrm{~mL}$ of the culture in liquid medium 79 (Fred and Waksman, 1928) with strains at log growth stage $\left(10^{9}\right.$ cells $\left.\mathrm{mL}^{-1}\right)$. In treatments 
Table 1 - Number of nodules (NN), nodules dry matter (NDM), shoot dry matter (SDM), root dry matter (RDM), and total dry matter (TDM) of bean plants inoculated with strains isolated from the Amazon pastureland.

\begin{tabular}{|c|c|c|c|c|c|}
\hline Treatment & NN & NDM & SDM & RDM & TDM \\
\hline & NN per plant & & - g per & plant - & \\
\hline UFLA 02-305 & $0.00 \mathrm{c}$ & $0.00 \mathrm{~b}$ & $0.10 \mathrm{c}$ & $0.09 c$ & $0.19 c$ \\
\hline UFLA 02-306 & $0.00 \mathrm{c}$ & $0.00 \mathrm{~b}$ & $0.12 \mathrm{c}$ & $0.16 b$ & $0.28 \mathrm{c}$ \\
\hline UFLA 02-307 & $0.00 \mathrm{c}$ & $0.00 \mathrm{~b}$ & $0.15 c$ & $0.19 b$ & $0.34 \mathrm{c}$ \\
\hline UFLA 02-308 & $0.00 \mathrm{c}$ & $0.00 \mathrm{~b}$ & $0.16 \mathrm{c}$ & $0.15 b$ & $0.31 \mathrm{c}$ \\
\hline UFLA 02-309 & $0.00 \mathrm{c}$ & $0.00 \mathrm{~b}$ & $0.16 \mathrm{c}$ & $0.19 b$ & $0.36 c$ \\
\hline UFLA 02-310 & $0.00 \mathrm{c}$ & $0.00 \mathrm{~b}$ & $0.16 \mathrm{c}$ & $0.18 b$ & $0.35 c$ \\
\hline UFLA 02-311 & $0.00 \mathrm{c}$ & $0.00 \mathrm{~b}$ & $0.16 \mathrm{c}$ & $0.12 b$ & $0.29 c$ \\
\hline UFLA 02-272 & $0.00 \mathrm{c}$ & $0.00 \mathrm{~b}$ & $0.16 c$ & $0.23 \mathrm{a}$ & $0.40 c$ \\
\hline UFLA 02-295 & $0.00 \mathrm{c}$ & $0.00 \mathrm{~b}$ & $0.16 c$ & $0.23 a$ & $0.40 c$ \\
\hline UFLA 02-312 & $0.00 \mathrm{c}$ & $0.00 \mathrm{~b}$ & $0.17 c$ & $0.17 b$ & $0.34 \mathrm{c}$ \\
\hline UFLA 02-313 & $0.00 \mathrm{c}$ & $0.00 \mathrm{~b}$ & $0.17 c$ & $0.22 \mathrm{a}$ & $0.38 c$ \\
\hline UFLA 02-314 & $0.00 \mathrm{c}$ & $0.00 \mathrm{~b}$ & $0.17 c$ & $0.23 \mathrm{a}$ & $0.40 c$ \\
\hline UFLA 02-315 & $0.00 \mathrm{c}$ & $0.00 \mathrm{~b}$ & $0.18 c$ & $0.20 \mathrm{~b}$ & $0.38 c$ \\
\hline UFLA 02-316 & $0.00 \mathrm{c}$ & $0.00 \mathrm{~b}$ & $0.18 c$ & $0.16 b$ & $0.33 c$ \\
\hline UFLA 02-273 & $0.00 \mathrm{c}$ & $0.00 \mathrm{~b}$ & $0.19 c$ & $0.23 a$ & $0.42 c$ \\
\hline UFLA 02- 267 & $0.00 \mathrm{c}$ & $0.00 \mathrm{~b}$ & $0.19 c$ & $0.21 \mathrm{~b}$ & $0.39 c$ \\
\hline UFLA 02-317 & $0.00 \mathrm{c}$ & $0.00 \mathrm{~b}$ & $0.19 c$ & $0.20 \mathrm{~b}$ & $0.40 c$ \\
\hline UFLA 02-318 & $0.00 \mathrm{c}$ & $0.00 \mathrm{~b}$ & $0.19 c$ & $0.28 \mathrm{a}$ & $0.48 b$ \\
\hline UFLA 02-281 & $0.00 \mathrm{c}$ & $0.00 \mathrm{~b}$ & $0.20 c$ & $0.23 \mathrm{a}$ & $0.43 c$ \\
\hline UFLA 02-289 & $0.00 \mathrm{c}$ & $0.00 \mathrm{~b}$ & $0.20 c$ & $0.18 b$ & $0.38 c$ \\
\hline UFLA 02-269 & $0.00 \mathrm{c}$ & $0.00 \mathrm{~b}$ & $0.20 c$ & $0.20 \mathrm{~b}$ & $0.40 c$ \\
\hline UFLA 02-319 & $0.00 \mathrm{c}$ & $0.00 \mathrm{~b}$ & $0.21 \mathrm{c}$ & $0.25 \mathrm{a}$ & $0.45 b$ \\
\hline UFLA 02-290 & $0.00 \mathrm{c}$ & $0.00 \mathrm{~b}$ & $0.21 \mathrm{c}$ & $0.23 a$ & $0.43 c$ \\
\hline UFLA 02-272 & $0.00 \mathrm{c}$ & $0.00 \mathrm{~b}$ & $0.21 \mathrm{c}$ & $0.21 \mathrm{~b}$ & $0.41 \mathrm{c}$ \\
\hline UFLA 02-320 & $0.00 \mathrm{c}$ & $0.00 \mathrm{~b}$ & $0.21 \mathrm{c}$ & $0.14 \mathrm{~b}$ & $0.35 c$ \\
\hline UFLA 02-283 & $0.00 \mathrm{c}$ & $0.00 \mathrm{~b}$ & $0.21 \mathrm{c}$ & $0.31 \mathrm{a}$ & $0.52 b$ \\
\hline UFLA 02-293 & $0.00 \mathrm{c}$ & $0.00 \mathrm{~b}$ & $0.21 \mathrm{c}$ & $0.22 \mathrm{a}$ & $0.41 \mathrm{c}$ \\
\hline UFLA 02-271 & $0.00 \mathrm{c}$ & $0.00 \mathrm{~b}$ & $0.21 \mathrm{c}$ & $0.19 b$ & $0.40 c$ \\
\hline UFLA 02-284 & $0.00 \mathrm{c}$ & $0.00 \mathrm{~b}$ & $0.21 \mathrm{c}$ & $0.26 \mathrm{a}$ & $0.47 b$ \\
\hline UFLA 02-277 & $0.00 \mathrm{c}$ & $0.00 \mathrm{~b}$ & $0.21 \mathrm{c}$ & $0.25 a$ & $0.46 b$ \\
\hline UFLA 02-295 & $0.00 \mathrm{c}$ & $0.00 \mathrm{~b}$ & $0.22 c$ & $0.19 b$ & $0.42 c$ \\
\hline UFLA 02-280 & $15.21 \mathrm{~b}$ & 0.014 a & $0.22 c$ & $0.19 b$ & $0.46 b$ \\
\hline UFLA 02-270 & $0.00 \mathrm{c}$ & $0.00 \mathrm{~b}$ & $0.22 c$ & $0.24 \mathrm{a}$ & $0.46 b$ \\
\hline UFLA 02-278 & $0.00 \mathrm{c}$ & $0.00 \mathrm{~b}$ & $0.22 c$ & $0.24 \mathrm{a}$ & $0.47 b$ \\
\hline UFLA 02-287 & $12.04 \mathrm{~b}$ & $0.013 a$ & $0.23 c$ & $0.19 b$ & $0.42 c$ \\
\hline UFLA 02-302 & $0.00 \mathrm{c}$ & $0.00 \mathrm{~b}$ & $0.23 c$ & $0.21 \mathrm{~b}$ & $0.44 b$ \\
\hline UFLA 02-211 & $0.00 \mathrm{c}$ & $0.00 \mathrm{~b}$ & $0.23 c$ & $0.27 \mathrm{a}$ & $0.50 \mathrm{~b}$ \\
\hline UFLA 02-303 & $0.00 \mathrm{c}$ & $0.00 \mathrm{~b}$ & $0.24 \mathrm{c}$ & $0.22 \mathrm{a}$ & $0.46 b$ \\
\hline UFLA 02-276 & $0.00 \mathrm{c}$ & $0.00 \mathrm{~b}$ & $0.24 \mathrm{c}$ & $0.23 a$ & $0.48 b$ \\
\hline UFLA 02-292 & $0.00 \mathrm{c}$ & $0.00 \mathrm{~b}$ & $0.23 c$ & $0.24 \mathrm{a}$ & $0.50 \mathrm{~b}$ \\
\hline UFLA 02-301 & $0.00 \mathrm{c}$ & $0.00 \mathrm{~b}$ & $0.25 b$ & $0.25 a$ & $0.50 \mathrm{~b}$ \\
\hline UFLA 02-15 & $0.00 \mathrm{c}$ & $0.00 \mathrm{~b}$ & $0.25 b$ & $0.23 \mathrm{a}$ & $0.49 b$ \\
\hline UFLA 02-296 & $0.00 \mathrm{c}$ & $0.00 \mathrm{~b}$ & $0.25 b$ & $0.28 a$ & $0.51 b$ \\
\hline UFLA 02-274 & $0.00 \mathrm{c}$ & $0.00 \mathrm{~b}$ & $0.25 b$ & $0.23 a$ & $0.47 b$ \\
\hline UFLA 02-299 & $0.00 \mathrm{c}$ & $0.00 \mathrm{~b}$ & $0.26 b$ & $0.21 \mathrm{a}$ & $0.47 b$ \\
\hline UFLA 02-298 & $0.00 \mathrm{c}$ & $0.00 \mathrm{~b}$ & $0.26 \mathrm{~b}$ & $0.23 a$ & $0.50 \mathrm{~b}$ \\
\hline UFLA 02-300 & $0.00 \mathrm{c}$ & $0.00 \mathrm{~b}$ & $0.27 b$ & $0.29 a$ & $0.56 b$ \\
\hline UFLA 02-09 & $11.45 \mathrm{~b}$ & $0.017 \mathrm{a}$ & $0.21 \mathrm{c}$ & $0.21 \mathrm{~b}$ & $0.42 c$ \\
\hline UFLA 02-286 & $0.00 \mathrm{c}$ & $0.00 \mathrm{~b}$ & $0.27 b$ & $0.22 a$ & $0.49 b$ \\
\hline UFLA 02-282 & $0.00 \mathrm{c}$ & $0.00 \mathrm{~b}$ & $0.28 b$ & $0.24 \mathrm{a}$ & $0.51 b$ \\
\hline UFLA 02-268 & $0.00 \mathrm{c}$ & $0.00 \mathrm{~b}$ & $0.20 \mathrm{c}$ & $0.23 a$ & $0.51 b$ \\
\hline
\end{tabular}

\begin{tabular}{lrllll} 
UFLA 02-279 & $18.66 \mathrm{a}$ & $0.016 \mathrm{a}$ & $0.29 \mathrm{~b}$ & $0.21 \mathrm{~b}$ & $0.50 \mathrm{~b}$ \\
UFLA 02-285 & $11.35 \mathrm{~b}$ & $0.015 \mathrm{a}$ & $0.28 \mathrm{~b}$ & $0.21 \mathrm{~b}$ & $0.49 \mathrm{~b}$ \\
UFLA 02-275 & $0.00 \mathrm{c}$ & $0.00 \mathrm{~b}$ & $0.31 \mathrm{~b}$ & $0.15 \mathrm{~b}$ & $0.46 \mathrm{~b}$ \\
UFLA 02-288 & $0.00 \mathrm{c}$ & $0.00 \mathrm{~b}$ & $0.31 \mathrm{~b}$ & $0.26 \mathrm{a}$ & $0.57 \mathrm{a}$ \\
UFLA 02-297 & $0.00 \mathrm{c}$ & $0.00 \mathrm{~b}$ & $0.34 \mathrm{~b}$ & $0.22 \mathrm{a}$ & $0.56 \mathrm{~b}$ \\
Control 52.5 mg L-1 & $0.00 \mathrm{c}$ & $0.00 \mathrm{~b}$ & $0.55 \mathrm{a}$ & $0.36 \mathrm{a}$ & $0.92 \mathrm{a}$ \\
Control 5.25 mg L-1 & $0.00 \mathrm{c}$ & $0.00 \mathrm{~b}$ & $0.16 \mathrm{c}$ & $0.17 \mathrm{~b}$ & $0.33 \mathrm{c}$ \\
\hline $\mathrm{CV} \%$ & 48.74 & 0.35 & 28.51 & 22.95 & 21.35
\end{tabular}

$\overline{\mathrm{CV}}=$ coefficient of variation. Means followed by the same letters in the columns do not differ by the Scott-Knott test at $5 \%$ probability.

without inoculation, only $1 \mathrm{~mL}$ autoclaved liquid culture medium 79 was added.

After sowing and inoculating, jars were covered with a layer of sand paraffin $(10 \mathrm{~kg}$ sand, $1 \mathrm{~L}$ chloroform, and $10 \mathrm{~g}$ paraffin) to prevent contamination. Thinning was carried out five days after emergence, leaving one plant per jar. During the experiment, the nutrient solution was prepared, autoclaved, and periodically replaced in jars, according to the plant absorption rate.

At 45 days after sowing, corresponding to the flowering stage, plants were harvested to determine the following traits: number of nodules $(\mathrm{NN})$; nodules dry matter (NDM); shoot dry matter (SDM); root dry matter (RDM); total dry matter (TDM); and $\mathrm{N}$ accumulation in the shoots (NAS). To determine NDM and SDM, the same procedures described for the authentication experiment were used. After weighing, $\mathrm{N}$ accumulated in the shoots (NAS) was calculated by multiplying the weight of dry shoots by the $\mathrm{N}$ content, and measured by the semi micro-Kjeldahl method described by Liao (1981).

Data were subjected to analysis of variance, using the statistical program SISVAR, version 5.1 (Ferreira, 2011). Treatments were compared by the Scott-Knott test at $5 \%$ probability. NDM and NN values had been previously transformed into square root of $(\mathrm{Y}+0.5)$.

In order to confirm the cultural and genetic characteristics of the inoculated strains, 10 nodules were selected from each co-inoculation treatment for re-isolation. For surface disinfection, nodules were first immersed in $95 \%$ ethanol. Next, in $\mathrm{H}_{2} \mathrm{O}_{2}$ for 3 min, and after that, they were rinsed 10 times with sterile distilled water (Guimarães et al., 2012). Subsequently, nodules were macerated in plates containing culture medium 79 (Fred and Waksman, 1928) and the material was spread to obtain isolated colonies. Afterward, sequencing was carried out as described before.

\section{Results}

Of the 56 strains studied, 35 were successfully identified. A comparison of the 16S rRNA gene partial sequencing of strains evaluated with sequences deposited in the GenBank revealed that strains belong to Rhizobium, Burkholderia, Xanthomonas, Brevibacillus, and Bacillus genera, with predominance of Pseudomonas (Table 2). 
Table 2 - Identification of strains isolated from nodules of common bean grown in soil of pasture land from Amazon based on the most similar sequences found in GenBank (NCBI).

\begin{tabular}{|c|c|c|c|c|}
\hline \multirow{2}{*}{ Strains } & \multirow{2}{*}{ Sequence length } & \multicolumn{3}{|c|}{ Most similar sequences found in GenBank (NCBI) } \\
\hline & & Similarity & Accession number & Species \\
\hline & & $\%$ & & \\
\hline UFLA 02-267 & 612 & 99 & KF295815 & Burkholderia fungorum \\
\hline UFLA 02-268 & 714 & 100 & FJ534682 & Burkholderia sp. \\
\hline UFLA 02-288 & 783 & 100 & HG794272 & Burkholderia fungorum \\
\hline UFLA 02-294 & 1340 & 99 & CP010027 & Burkholderia fungorum \\
\hline UFLA 03-304 & 1308 & 99 & СР010027 & Burkholderia fungorum \\
\hline UFLA 02-302 & 1274 & 99 & СР010027 & Burkholderia fungorum \\
\hline UFLA 02-270 & 1244 & 100 & LC005608 & Brevibacillus sp. \\
\hline UFLA 02-295 & 1260 & 99 & LC005608 & Brevibacillus sp. \\
\hline UFLA 02-296 & 904 & 99 & KT580607 & Brevibacillus sp. \\
\hline UFLA 02-299 & 1305 & 99 & LC005608 & Brevibacillus sp. \\
\hline UFLA 02-290* & 985 & 100 & NR116873 & Bacillus magaterium \\
\hline UFLA 02-298* & 1001 & 99 & AB533761 & Bacillus sp. \\
\hline UFLA 02-15 & 792 & 100 & AY822513 & Bacterium RSD-1-2 \\
\hline UFLA 02-289 & 358 & 100 & KC894165 & Pseudomonas sp. \\
\hline UFLA 02-291 & 738 & 100 & KT154837 & Pseudomonas sp. \\
\hline UFLA 02-293* & 1329 & 100 & СР011789 & Pseudomonas putida \\
\hline UFLA 02-297 & 1324 & 99 & KJ748611 & Pseudomonas putida \\
\hline UFLA 02-303 & 498 & 99 & KP279906 & Pseudomonas sp. \\
\hline UFLA 02-286 & 601 & 100 & KU350602 & Pseudomonas sp. \\
\hline UFLA 02-281 * & 685 & 99 & LC270240 & Pseudomonas sp. \\
\hline UFLA 02-282* & 738 & 100 & LN847264 & Pseudomonas sp. \\
\hline UFLA 02-283 & 584 & 100 & JX827617 & Pseudomonas sp. \\
\hline UFLA 02-284 & 498 & 100 & KP067092 & Pseudomonas sp. \\
\hline UFLA 02-269 & 681 & 100 & KC879711 & Pseudomonas koreensis \\
\hline UFLA 02-273 & 501 & 100 & EU853182 & Pseudomonas sp. \\
\hline UFLA 02-275 & 374 & 100 & KM08802 & Pseudomonas sp. \\
\hline UFLA 02-276* & 369 & 100 & GQ868350 & Pseudomonas sp. \\
\hline UFLA 02-277 & 601 & 100 & KF053343 & Pseudomonas sp. \\
\hline UFLA 02-278 & 403 & 100 & KF767887 & Pseudomonas putida \\
\hline UFLA 02-279 & 530 & 100 & KP128829 & Rhizobium sp. \\
\hline UFLA 02-280 & 988 & 99 & KJ632048 & Rhizobium sp. \\
\hline UFLA 02-09 & 453 & 99 & KC113610 & Rhizobium etli \\
\hline UFLA 02-285 & 651 & 99 & KJ513849 & Rhizobium sp. \\
\hline UFLA 02-287 & 704 & 100 & KJ734011 & Rhizobium sp. \\
\hline UFLA 02-274* & 649 & 100 & KF923840 & Xanthomonas sp. \\
\hline
\end{tabular}

${ }^{*}$ Strains evaluated for growth promotion potential when subjected to inoculation and co-inoculation with CIAT 899 strain.

In the individual inoculation experiment (experiment 1), treatments influenced all the evaluated parameters $(p \leq 0.05)$ (Table 1$)$. There was no nodulation in the control groups, indicating no contamination in the experiment.

Of the 56 strains that were inoculated, only strains UFLA 02-09, UFLA 02-287, UFLA 02-279, UFLA 02-280 and UFLA 02-285 nodulated bean plants and belonged to the Rhizobium genus (Table 2). All strains showed SDM production lower $(p \geq 0.05)$ when compared to the control with high $\mathrm{N}$-mineral concentration (Table 1). Among the evaluated strains, 14 stood out for presenting SDM production superior to that of the control with low $\mathrm{N}$ concentration $(p \leq 0.05)$ (Table 1$)$. Of these strains, only two are nodulating strains (UFLA 02-279 and UFLA 02-285).
In relation to RDM, $55 \%$ of the strains had better performance, with gains similar $(p \leq 0.05)$ to that of the control with high $\mathrm{N}$ concentration (Table 1).

Seven strains studied in the inoculation and coinoculation tests (experiment 2) are non-nodulating species. However, they showed good results for plant growth promotion, as observed in experiment 1 . These strains belong to the genera: Pseudomonas (UFLA 02276, UFLA 02-281, UFLA 02-282, UFLA 02-293), Bacillus (UFLA 02-290 and UFLA 02-298) and Xanthomonas (UFLA 02-274).

There was significant interaction between inoculation of the seven strains and forms of $\mathrm{N}$ supply for all variables studied, except for NDM (Tables 3 and 4), since only the forms of $\mathrm{N}$ supply presented effects. 
Table 3 - Number of nodules, nodules dry matter, shoot dry matter, root dry matter, and total dry matter of bean plants inoculated with strains isolated from Amazon pastureland in different forms of $\mathrm{N}$ supply.

\begin{tabular}{|c|c|c|c|c|}
\hline \multicolumn{5}{|c|}{ Number of nodules (NN per plant) } \\
\hline Strains & Species & CIAT 899 & High $\mathrm{N}$ concentration $\left(52.5 \mathrm{mg} \mathrm{L}^{-1}\right)$ & Low $\mathrm{N}$ concentration $\left(5.25 \mathrm{mg} \mathrm{L}^{-1}\right)$ \\
\hline UFLA 02-282 & Pseudomonas sp. & $42.33 \mathrm{dA}$ & $0.00 \mathrm{aB}$ & $0.00 \mathrm{aB}$ \\
\hline UFLA 02-276 & Pseudomonas sp. & $44.33 \mathrm{dA}$ & $0.00 \mathrm{aB}$ & $0.00 \mathrm{aB}$ \\
\hline UFLA 02-290 & Bacillus magaterium & $120.33 \mathrm{bA}$ & $0.00 \mathrm{aB}$ & $0.00 \mathrm{aB}$ \\
\hline UFLA 02-281 & Pseudomonas sp. & $90.00 \mathrm{cA}$ & $0.00 \mathrm{aB}$ & $0.00 \mathrm{aB}$ \\
\hline UFLA 02-298 & Bacillus sp. & $187.00 \mathrm{aA}$ & $0.00 \mathrm{aB}$ & $0.00 \mathrm{aB}$ \\
\hline UFLA 02-293 & Pseudomonas putida & $176.33 \mathrm{aA}$ & $0.00 \mathrm{aB}$ & $0.00 \mathrm{aB}$ \\
\hline UFLA 02-274 & Xanthomonas sp. & $85.66 \mathrm{cA}$ & $0.00 \mathrm{aB}$ & $0.00 \mathrm{aB}$ \\
\hline Control & & $86.00 \mathrm{cA}$ & $0.00 \mathrm{aB}$ & $0.00 \mathrm{aB}$ \\
\hline \multicolumn{5}{|c|}{ Shoot dry matter (g per plant) } \\
\hline UFLA 02-282 & Pseudomonas sp. & $0.34 \mathrm{bB}$ & $2.48 \mathrm{aA}$ & $0.26 \mathrm{bB}$ \\
\hline UFLA 02-276 & Pseudomonas sp. & $0.39 \mathrm{bA}$ & $0.48 \mathrm{cA}$ & $0.27 \mathrm{bA}$ \\
\hline UFLA 02-290 & Bacillus magaterium & $0.45 b B$ & $1.41 \mathrm{bA}$ & $0.33 \mathrm{bB}$ \\
\hline UFLA 02-281 & Pseudomonas sp. & $1.06 \mathrm{aA}$ & $1.17 \mathrm{bA}$ & $0.53 \mathrm{bB}$ \\
\hline UFLA 02-298 & Bacillus sp. & $1.37 \mathrm{aB}$ & $2.08 \mathrm{aA}$ & $0.87 \mathrm{aB}$ \\
\hline UFLA 02-293 & Pseudomonas putida & $1.67 \mathrm{aA}$ & $2.13 \mathrm{aA}$ & $0.49 \mathrm{bB}$ \\
\hline UFLA 02-274 & Xanthomonas sp. & $0.40 \mathrm{bB}$ & $1.50 \mathrm{bA}$ & $0.29 \mathrm{bB}$ \\
\hline Control & & $0.19 \mathrm{bB}$ & $0.92 \mathrm{bA}$ & $0.17 \mathrm{bB}$ \\
\hline \multicolumn{5}{|c|}{ Root dry matter (g per plant) } \\
\hline UFLA 02-282 & Pseudomonas sp. & $0.26 \mathrm{aB}$ & $1.35 \mathrm{aA}$ & $0.32 \mathrm{aB}$ \\
\hline UFLA 02-276 & Pseudomonas sp. & $0.32 \mathrm{aA}$ & $0.48 \mathrm{cA}$ & $0.34 \mathrm{aA}$ \\
\hline UFLA 02-290 & Bacillus magaterium & $0.44 \mathrm{aA}$ & $0.57 \mathrm{cA}$ & $0.41 \mathrm{aA}$ \\
\hline UFLA 02-281 & Pseudomonas sp. & $0.42 \mathrm{aB}$ & $1.43 \mathrm{aA}$ & $0.58 \mathrm{aB}$ \\
\hline UFLA 02-298 & Bacillus sp. & $0.49 \mathrm{aB}$ & $1.05 \mathrm{bA}$ & $0.64 \mathrm{aB}$ \\
\hline UFLA 02-293 & Pseudomonas putida & $0.59 \mathrm{aB}$ & $1.05 \mathrm{bA}$ & $0.50 \mathrm{aB}$ \\
\hline UFLA 02-274 & Xanthomonas sp. & $0.34 \mathrm{aB}$ & $0.80 \mathrm{cA}$ & $0.38 \mathrm{aB}$ \\
\hline Control & & $0.50 \mathrm{aA}$ & $0.36 c B$ & $0.37 \mathrm{aB}$ \\
\hline \multicolumn{5}{|c|}{ Total dry matter (g per plant) } \\
\hline UFLA 02-282 & Pseudomonas sp. & $0.60 \mathrm{cB}$ & $3.84 \mathrm{aA}$ & $0.58 \mathrm{bB}$ \\
\hline UFLA 02-276 & Pseudomonas sp. & $0.71 \mathrm{cA}$ & $0.96 \mathrm{cA}$ & $0.61 \mathrm{bA}$ \\
\hline UFLA 02-290 & Bacillus magaterium & $0.77 \mathrm{cA}$ & $0.87 \mathrm{cA}$ & $1.99 \mathrm{aA}$ \\
\hline UFLA 02-281 & Pseudomonas sp. & $1.48 \mathrm{bB}$ & $2.61 \mathrm{bA}$ & $1.11 \mathrm{bB}$ \\
\hline UFLA 02-298 & Bacillus sp. & $1.87 \mathrm{bB}$ & $3.13 \mathrm{bA}$ & $1.51 \mathrm{aB}$ \\
\hline UFLA 02-293 & Pseudomonas putida & $2.75 \mathrm{aA}$ & $2.95 \mathrm{bA}$ & $1.00 \mathrm{bB}$ \\
\hline UFLA 02-274 & Xanthomonas sp. & $0.75 \mathrm{cB}$ & $2.30 \mathrm{bA}$ & $0.67 \mathrm{bB}$ \\
\hline Control & & $0.69 \mathrm{cB}$ & $1.30 \mathrm{bA}$ & $0.54 \mathrm{bB}$ \\
\hline \multicolumn{5}{|c|}{ Nitrogen accumulation in shoots (mg per plant) } \\
\hline UFLA 02-282 & Pseudomonas sp. & $11.52 \mathrm{bB}$ & $96.57 \mathrm{aA}$ & $8.00 \mathrm{aC}$ \\
\hline UFLA 02-276 & Pseudomonas sp. & $18.13 \mathrm{bB}$ & $26.98 \mathrm{dA}$ & $6.79 \mathrm{aC}$ \\
\hline UFLA 02-290 & Bacillus magaterium & $16.86 \mathrm{bB}$ & $73.26 \mathrm{bA}$ & $6.28 \mathrm{aC}$ \\
\hline UFLA 02-281 & Pseudomonas sp. & $18.86 \mathrm{bB}$ & $47.63 \mathrm{cA}$ & $13.31 \mathrm{aC}$ \\
\hline UFLA 02-298 & Bacillus sp. & $41.77 \mathrm{aB}$ & $59.52 \mathrm{bA}$ & $12.96 \mathrm{aC}$ \\
\hline UFLA 02-293 & Pseudomonas putida & $50.35 \mathrm{aB}$ & $78.20 \mathrm{bA}$ & $17.81 \mathrm{aC}$ \\
\hline UFLA 02-274 & Xanthomonas sp. & $10.44 \mathrm{bB}$ & $46.11 \mathrm{cA}$ & $6.55 \mathrm{aB}$ \\
\hline Control & & $15.80 \mathrm{bB}$ & $26.86 \mathrm{dA}$ & $7.95 \mathrm{aC}$ \\
\hline
\end{tabular}

Means followed by the same lowercase letter in the columns between the seven strains and control by uppercase letters in the lines between the forms of $\mathrm{N}$ supply do not differ by the Scott-Knott test at $5 \%$ probability.

Bean inoculant strain (CIAT 899) efficiently nodulated this species, and the uninoculated controls showed no nodulation, indicating that the experiment was not contaminated and that the experimental conditions were favorable to nodulation. In the treatments individually inoculated with the seven previously selected strains, no bean nodulation was observed in the presence of low and high $\mathrm{N}$-mineral concentration in the nutrient solution, as expected.

Co-inoculation of UFLA 02-293 (Pseudomonas sp.), UFLA 02-290 and UFLA 02-298 (Bacillus sp.) with CIAT 899 resulted in higher $\mathrm{NN}(176,120$ and 187 nodules per 
Table 4 - Nodules dry matter (NDM) of bean plants inoculated with strains isolated from Amazon pastureland in different forms of $\mathrm{N}$ supply.

\begin{tabular}{llc}
\hline Strains & \multicolumn{1}{c}{ Species } & $\begin{array}{c}\text { Dry matter of } \\
\text { nodules }\end{array}$ \\
\hline UFLA 02-282 & Pseudomonas sp. & $16^{\mathrm{NS}}$ \\
UFLA 02-276 & Pseudomonas sp. & 12 \\
UFLA 02-290 & Bacillus magaterium & 55 \\
UFLA 02-281 & Pseudomonas sp. & 23 \\
UFLA 02-298 & Bacillus sp. & 27 \\
UFLA 02-293 & Pseudomonas putida & 35 \\
UFLA 02-274 & Xanthomonas sp. & 16 \\
Control & & 25 \\
\hline Forms of N supply & \\
\hline CIAT 899 & $79 \mathrm{a}$ \\
High N concentration $\left(52.5 \mathrm{mg} \mathrm{L}^{-1}\right)$ & $0 \mathrm{~b}$ \\
Low N concentration $\left(5.25 \mathrm{mg} \mathrm{L}^{-1}\right)$ & $0 \mathrm{~b}$ \\
\hline
\end{tabular}

Means followed by the same lowercase letters in the rows do not differ according to the Scott-Knott test at $5 \%$ probability.

plant, respectively) in relation $(p \leq 0.05)$ to individual inoculation with CIAT 899 (86 nodules per plant) (Table 3). Co-inoculation of CIAT 899 with UFLA 02-276 and UFLA 02-282 reduced NN (Table 3).

For NDM, effects were observed only for N supply $(p \geq 0.05)$ (Table 4). Co-inoculation of the seven strains with CIAT 899 provided the best results for this variable.

The best results for SDM production ( $p \leq 0.05)$ were observed in the treatments inoculated with the UFLA 02-293, UFLA 02-298 and UFLA 02-282 in the presence of high $\mathrm{N}$-mineral concentration (Table 3), in the co-inoculation of CIAT 899 with these strains along together with the UFLA 02-281, and in the individual inoculation of UFLA 02-298 in the presence of low $\mathrm{N}$ concentration (Table 3). The co-inoculation of CIAT 899 with UFLA 02-281 and UFLA 02-293 showed that the production of shoot dry matter was higher than in treatments cultivated with low $\mathrm{N}$ concentration $(p \leq 0.05)$, and similar to treatments cultivated with high $\mathrm{N}$ concentration $(p \geq 0.05)$ (Table 3).

Inoculation of seven strains in the presence of low $\mathrm{N}$ concentration and co-inoculation of these strains with CIAT 899 did not affect root dry matter production $(p$ $\geq 0.05$ ) (Table 3). However, in the presence of high $\mathrm{N}$ concentration, inoculation with UFLA 02-282, UFLA 02281, UFLA 02-298 and UFLA 02-293 promoted higher RDM production $(p \leq 0.05)$ and these strains were allocated in groups superior to the control, which was not inoculated with any strain (Table 3). Forms of $\mathrm{N}$ supply in the treatments inoculated with UFLA 02-276 and 02290 UFLA did not influence RDM production. Higher RDM production was observed for the other strains inoculated in the presence of high $\mathrm{N}$ concentration (Table 3).

Co-inoculation of UFLA 02-281, UFLA 02-298 and UFLA 02-293 strains with CIAT 899 provided the high- est TDM production ( $p \leq 0.05$ ) (Table 3). However, in the presence of high $\mathrm{N}$ concentration, only UFLA 02-282 stood out ( $p \leq 0.05$ ) (Table 3$)$. The same form of $\mathrm{N}$ supply showed inhibitory effect $(p \leq 0.05)$ (Table 3$)$ for inoculation of UFLA 02-276 and UFLA 02-290 strains. The highest TDM production in the presence of low N concentration was verified by the inoculation with UFLA 02-290 and UFLA 02-298 ( $p \leq 0.05$ ) (Table 3). Treatments with high $\mathrm{N}$ concentration were superior to the other forms of $\mathrm{N}$ supply, except for the treatments with co-inoculation of CIAT 899 with UFLA 02-276, UFLA 02-290 and UFLA 02-293, with no difference between them ( $p \leq 0.05)$ (Table 3). Therefore, when inoculated with CIAT 899, these strains were effective in promoting bean growth, since inoculation with only CIAT 899 promoted TDM similar to the control.

Treatments with low $\mathrm{N}$ concentration showed that inoculation of the seven strains did not influence NAS. However, NAS increased when plants were cultivated in high $\mathrm{N}$ concentrations inoculated with UFLA 02-282, UFLA 02-290, UFLA 02-281, UFLA 02-298 UFLA 02-274 and UFLA 02-293, or with the co-inoculation of CIAT 899 with UFLA 02-298 and UFLA 02-293. Treatments inoculated in the presence of high $\mathrm{N}$ concentration promoted higher NAS. Co-inoculation results were higher than in treatments with low $\mathrm{N}$ concentration (Table 3).

Results of sequencing re-isolated strains (Table 5) suggested that four are endophytes nodules. Re-isolation of UFLA 02-274 (Xanthomonas sp.) and UFLA 02-290 (Bacillus sp.) (Table 2) was not successful. Thus, their identities were not possible to confirm. UFLA 02-298 was not successfully amplified and was only evaluated by phenotypic characterization in culture medium. CIAT 899 was also observed, confirming that nodulation was promoted by the bean inoculant strain.

\section{Discussion}

The 16S rRNA gene sequencing, with the identification of six distinct genera, demonstrate a significant incidence of possible endophytes nodules, since most genera are not recognized as legume symbiont.

Among the studied strains, bacteria of the genus Rhizobium were the only ones to nodulate bean plants. Other studies report nodulation of this bean species by $R$. tropici (Martínez-Romero et al., 1991), R. etli (Segovia et al.,1993; Wang et al., 1999), $R$. gallicum (Amarger et al., 1997), R. giardinii (Amarger et al., 1997), R. yanglingense (Tan et al., 2001) and by other genera, such as Sinorhizobium sp. (Toledo et al., 2003), Mesorhizobium sp. (Chen et al., 1991) and Burkholderia sp. (Ferreira et al., 2012).

PGPR can be free-living, associative or endophytic. Endophytic PGPR are able to colonize plant roots and, in the case of legume species, it is capable of cohabiting with BNF within the nodules. According to Kan et al. (2007), these bacteria possibly penetrate into the plant tissue, together with nodulating strains, during infection 
Table 5 - Genetic identification of Re-isolated strains from nodules of common bean obtained from co-inoculation experiment based on the most similar sequences found in GenBank (NCBI).

\begin{tabular}{lcccc}
\hline $\begin{array}{l}\text { Re-isolated } \\
\text { strains }\end{array}$ & $\begin{array}{c}\text { Sequence } \\
\text { length }\end{array}$ & \multicolumn{3}{c}{$\begin{array}{c}\text { Most similar sequences found in GenBank } \\
\text { (NCBI) }\end{array}$} \\
\cline { 3 - 6 } & & Similarity & $\begin{array}{l}\text { Accession } \\
\text { number }\end{array}$ & \multicolumn{1}{c}{ Species } \\
\hline UFLA 02-276 & 403 & 100 & GQ868350 & Pseudomonas sp. \\
UFLA 02-276 & 1291 & 100 & GQ868350 & Pseudomonas sp. \\
UFLA 02-276 & 812 & 99 & GQ868350 & Pseudomonas sp. \\
UFLA 02-276 & 1014 & 100 & GQ868350 & Pseudomonas sp. \\
UFLA 02-281 & 476 & 100 & LC270240 & Pseudomonas sp. \\
UFLA 02-281 & 614 & 100 & LC270240 & Pseudomonas sp. \\
UFLA 02-281 & 944 & 100 & LC270240 & Pseudomonas sp. \\
UFLA 02-281 & 960 & 99 & LC270240 & Pseudomonas sp. \\
UFLA 02-281 & 1220 & 100 & LC270240 & Pseudomonas sp. \\
UFLA 02-293 & 654 & 100 & CP011789 & Pseudomonas putida \\
UFLA 02-282 & 895 & 100 & LN847264 & Pseudomonas putida \\
UFLA 02-282 & 1327 & 99 & LN847264 & Pseudomonas putida \\
CIAT899 Control & 88 & 100 & KF773126 & Rhizobium tropici \\
CIAT 899 Control & 627 & 100 & KF773126 & Rhizobium tropici \\
CIAT 899 T3 & 840 & 100 & KF773126 & Rhizobium tropici \\
CIAT 899 T3 & 564 & 100 & KF773126 & Rhizobium tropici \\
CIAT 899 T1R1 & 802 & 99 & KT356836 & Rhizobium sp. \\
CIAT 899 T1R2 & 1221 & 99 & NR102511 & Rhizobium sp. \\
CIAT 899 T1R3 & 899 & 100 & KP205042 & Rhizobium sp. \\
CIAT 899 T1R3 & 1166 & 100 & LN833444 & Rhizobium sp. \\
CIAT 899 T3R3 & 951 & 99 & KP760691 & Rhizobium sp. \\
CIAT 899 T18R3 & 1013 & 100 & KF773126 & Rhizobium tropici \\
CIAT 899 T18R4 & 865 & 99 & KF773126 & Rhizobium tropici \\
CIAT 899 T18R1 & 1223 & 100 & NR102511 & Rhizobium sp. \\
CIAT 899 T18R2 & 654 & 100 & KF773126 & Rhizobium tropici \\
\hline & & & & \\
\hline
\end{tabular}

and nodules formation. The genera Agrobacterium, Pseudomonas, Enterobacter, Pantoea, Bacillus and Paenibacillus are more frequently reported as nodule endophytes (Kan et al., 2007; Li et al., 2008; Shiraishi et al., 2010; Costa et al., 2016). In this study, Bacillus (UFLA 02-298) and Paenibacillus (UFLA 02-276, UFLA 02-293, UFLA 02-281 and UFLA 02-282) were detected as possible endophytes nodules.

Similar to pasturelands, a significant genetic diversity of nodulating and non nodulating PGPR genera were also found in soils under agricultural systems in western Amazon by Guimarães et al. (2012) (Bradyrhizobium, Rhizobium, Burkholderia and Achromobacter) and agroforestry systems by Jaramillo et al. (2013) (Bradyrhizobium, Rhizobium, Ochrobactrum, Paenibacillus, Bosea, Bacillus, Enterobacter, Stenotrophomonas) using Vigna unguiculata (L.) Walp as trap plant. These results show that high symbiotic and genetic diversity of bacterial strains from different land use systems in the western Amazon as a potential PGPR source.

In the first experiment, although strains belonging to the genera Pseudomonas, Xanthomonas, Burkholderia, and Bacillus (Table 2) did not nodulate common bean plants, they promoted plant growth in low $\mathrm{N}$ concentration. This result was probably caused due to phytoestimulation by other biological processes, such as phytohormones production (Costa et al., 2016). Azospirillum, Bacillus, Enterobacter, Herbaspirillum, Paenibacillus, Pseudomonas and Burkholderia strains are often described as potential plant growth promoters due to the action in different biological processes, especially in phosphate solubilization and phytohormones synthesis (particularly IAA) (Samavat et al., 2012; Oliveira-Longatti et al., 2013; 2014; Costa et al., 2016).

The ability to fix $\mathrm{N}$ may be affected by several biotic and abiotic factors (Tsai, 1993; Ali et al., 2009). The interaction of Rhizobium with other microorganisms in the soil is one of the factors that affects this process and both stimulation and inhibition of nodulation and plant growth may occur, depending on the interaction between symbionts and growth-promoting bacterial strains. In the present study, this fact was presented by nodulation stimulation by Pseudomonas sp. (UFLA 02-293), Bacillus sp. (UFLA 02-290) and Pseudomonas sp. (UFLA 02-298) strains, and nodulation inhibition by Pseudomonas sp. (UFLA 02-282 and UFLA 276) strains. However, not all strains that stimulated nodulation in co-inoculated treatments with CIAT 899 promoted growth or increased SDM, RDM, and TDM production.

In RDM, SDM and TDM production, NAS was different for strains depending on $\mathrm{N}$ supply, except for RDM in the co-inoculated treatments and in the presence of low $\mathrm{N}$ concentration. Notably, the highest increase in SDM was obtained by the combination of Bacillus sp. (UFLA 02-298) and Paenibacillus sp. (UFLA 02-293, UFLA 02-281 and UFLA 02-282) co-inoculated with Rhizobium tropici CIAT 899. Individual inoculations of PGPR in the presence of low $\mathrm{N}$ concentration did not influence NAS. This result was expected since they are not nodulating strains, nor $\mathrm{N}_{2}$-fixing strains; however, they influence plant growth by other processes (Costa et al., 2016). Nevertheless, co-inoculation of PGPR with Bacillus sp. (UFLA 02-298) and Paenibacillus sp. (02-293) together with Rhizobium tropici (CIAT 899) increased NAS.

In the literature, PGPR of the genus Pseudomonas are reported as growth promoters, as verified by Samatava et al. (2012). This genus also showed relevant performance when co-inoculated with Rhizobium tropici CIAT 899. In other studies, the contribution of the co-inoculation of Paenibacillus strains (Rodrigues et al., 2012) and Enterobacter (Costa et al., 2016) with Bradyrhizobium was observed on the dry matter yield of cowpea and soybean plants, respectively.

Inoculation of CIAT 899 with Pseudomonas sp. and Bacillus sp. can be an effective strategy to produce bio-fertilizers for beans. The results help establish an inoculum or a combination of inoculum for bean yield improvement, as well as the understanding of their performance under low and high $\mathrm{N}$-minerals concentrations. 


\section{Conclusions}

$16 \mathrm{~S}$ rRNA gene sequencing showed the predominance of Pseudomonas genus as bean plants nodules endophytic strains. Other genera were identified: Rhizobium, Burkholderia, Xanthomonas and Bacillus. Thus, high incidence of possible endophytic strains was found in the nodules, since most of these genera are not known as legume plants symbiont.

This result also demonstrates a significant genetic diversity of bacterial strains under pasture system by the presence of strains of Bacillus and Pseudomonas genera, showing potential to be used as plant growth promoters. Inoculation of seven strains with CIAT 899 promoted varied plant growth in the different forms of $\mathrm{N}$ supply. N-mineral supply may be replaced by the coinoculation of CIAT 899 with UFLA 02-281, UFLA 02286 and UFLA 02-293, which are plant growth promoting strains. Tests under field conditions are necessary to validate the promising results on a large scale.

\section{Acknowledgments}

The authors thank the Brazilian National Council for Scientific and Technological Development (CNPq) 304527/2016-5; 560551/2010-0; 106350/20153; 141672/2014-5; Coordination for the Improvement of Higher Level Personnel (CAPES) - PROEX AUXPE 5902014 and Minas Gerais State Foundation for Research Support Support (FAPEMIG) - PACCSS (Programme for supporting graduate courses with grades six and seven) AUXPE 26182012 for financial support and for granting and fellowships

\section{References}

Ali, S.F.; Rawat, L.S.; Meghvansi, M.K.; Mahna, S.K. 2009. Selection of stress-tolerant rhizobial isolates of wild legumes growing in dry regions of Rajasthan, India. Journal of Agriculture and Biological Sciences 4: 13-18.

Amarger, N.; Macheret, V.; Laguerre, G. 1997. Rhizobium gallicum sp. nov. and Rhizobium giardinii sp. nov. from Phaseolus vulgaris nodules. International Journal of Systematic Bacteriology 47: 996-1006.

Carvalho, T.S.; Jesus, E.C.; Barlow, J.; Gardner, T.A.; Soares, I.C.; Tiedje, J.M.; Moreira, F.M.S. 2016. Land use intensification in the humid tropics increased both alpha and beta diversity of soil bacteria. Ecology 97: 2760-2771.

Chen, W.X.; Li, Y.L.; Wang, E.T.; Yuan, H.L.; Li, J.L. 1991. Rhizobium huahuii sp. nov. isolated from root nodules of Astragalus sinicus. International Journal of Systematic Bacteriology 41: 275-280.

Costa, E.M.; Carvalho, F.; Nóbrega, R.S.A.; Silva J.S.; Moreira, F.M.S. 2016. Bacterial strains from floodplain soils perform different plant-growth promoting processes and enhance cowpea growth. Scientia Agricola 73: 301-310.

Ferreira, D.F. 2011. Sisvar: a computer statistical analysis system. Ciência e Agrotecnologia 35: 1039-1042.
Ferreira, P.A.A.; Bomfeti, C.A.; Soares, B.L.; Moreira, F.M.S. 2012. Efficient nitrogen-fixing Rhizobium strains isolated from Amazonian soils are highly tolerant to acidity and aluminium. World Journal of Microbiology and Biotechnology 28: 19471959.

Fred, E.B.; Waksman, S.A. 1928. Laboratory Manual of General Microbiology: with Special Reference to the Microorganisms of the Soil. McGraw-Hill, New York, NY, USA.

Hoagland, D.R.; Arnon, D.T. 1950. The Water Culture Method for Growing Plants without Soil. California Agriculture Experiment Station, Berkeley, CA, USA.

Guimarães, A.A.; Jaramillo, P.M.D.; Nóbrega, R.S.A.; Florestino, L.A.; Silva, K.; Moreira, F.M.S. 2012. Genetic and symbiotic diversity of nitrogen fixing bacteria isolated from agricultural soils in the western Amazon by using cowpea as the trap plant. Applied and Environmental Microbiology 78: 6726-6733.

Jaramillo, P.M.D.; Guimarães, A.A.; Florentino, L.A.; Silva, K.B.; Nóbrega, R.S.A.; Moreira, F.M.S. 2013. Symbiotic nitrogen-fixing bacterial populations trapped from soils under agroforestry systems in the western Amazon. Scientia Agricola 70: 397-404.

Kan, F.L.; Chen, Z.Y.; Wang, E.T.; Tian, C.F.; Sui, X.H.; Chen, W.X. 2007. Characterization of symbiotic and endophytic bacteria isolated from root nodules of herbaceous legumes cultivated in Qinghai-Tibet plateau and in other zones of China. Archives of Microbiology 188: 103-115.

Li, J.H.; Wang, E.T.; Chena, W.F.; Chena, W.X. 2008. Genetic diversity and potential for promotion of plant growth detected in nodule endophytic bacteria of soybean cultivated in Heilongjiang province of China. Soil Biology and Biochemistry 40: 238-246.

Liao, C.F.H. 1981. Devarda's allow methods for total nitrogen determination. Soil Science Society of America Journal 45: 852855.

Marra, L.M.; Soares, C.R.F.S.; Oliveira, S.M.; Ferreira, P.A.A.; Soares, B.L.; Carvalho, R.F.; Lima, J.M.; Moreira, F.M.S. 2012. Biological nitrogen fixation and phosphate solubilization by bacteria isolated from tropical soils. Plant and Soil 357: 289-307.

Martínez-Romero, E.; Segovia, E.; Mercante, F.M.; Franco, A.A.; Graham, P.H.; Pardo, M.A. 1991. Rhizobium tropici, a novel species nodulating Phaseolus vulgaris L beans and Leucaena sp. trees. International Journal of Systematic Bacteriology 41: 417-426.

Medeiros, F.H.V.; Souza, R.M.; Medeiros, F.C.L.; Zhang, H.; Wheeler, T.; Payton, P.; Ferro, H.M.; Paré, P.W. 2011. Transcriptional profiling in cotton associated with Bacillus subtilis (UFLA285) induced biotic-stress tolerance. Plant and Soil 1-11.

Mishra, P.K.; Bisht, S.C.; Ruwari, P.; Joshi, G.K.; Singh, G.; Bisht, J.K.; Bhatt, J.C. 2011. Bioassociative effect of cold tolerant Pseudomonas spp. and Rhizobiu leguminosarum-PR1 on iron acquisition, nutrient uptake and growth of lentil (Lens culinaris L.). European Journal of Soil Biology 47: 35-43.

Moreira, F.M.S.; Nóbrega, R.S.A.; Jesus, E.C.; Ferreira, D.F.; Pérez, D.V. 2009. Differentiation in the fertility of Inceptisols as related to land use in the upper Solimões river region, western Amazon. Science of the Total Environment 408: 349-355. 
Niemann, S.; Puehler, A.; Tichy, H.V.; Simon, R.; Selbitshka, W. 1977. Evaluation of the resolving power of three different DNA fingerprinting methods to discriminate among isolates of a natural Rhizobium meliloti population. Journal of Applied Microbiology 82: 477-484.

Oliveira-Longatti, S.M.; Marra, L.M.; Moreira, F.M.S. 2013. Evaluation of plant growth-promoting traits of Burkholderia and Rhizobium strains isolated from Amazon soils for their coinoculation in common bean. African Journal of Microbiology Research 7: 948-959.

Oliveira-Longatti, S.M.; Marra, L.M.; Soares, B.L.; Bomfeti, C.A.; Silva, K.; Ferreira, P.A.V.; Moreira, F.M.S. 2014. Bacteria isolated from soils of the western Amazon and from rehabilitated bauxite-mining areas have potential as plant growth promoters. World Journal of Microbiology Biotechnology 30: 1239-1250.

Samavat, S.; Samavat, S.; Mafakheri, S.; Shakouri, M.J. 2012. Promoting common bean growth and nitrogen fixation by the co-inoculation of Rhizobium and Pseudomonas fluorescens isolates. Bulgarian Journal of Agricultural Science 18: 387-395.

Segovia, L.; Young, J.P.W.; Martínez-Romero, E. 1993. Reclassification of American Rhizobium leguminosarum biovar phaseoli type I strains as Rhizobium etli sp. Internatinal Journal of Systematic Bacteriology 43: 374-377.

Shiraishi, A.; Matsushita, N.; Hougetsu, T. 2010. Nodulation in black locust by the Gammaproteobacteria Pseudomonas sp. and the Betaproteobacteria Burkholderia sp. Systematic and Applied Microbiology. 33: 269-274.

Silva, K.; Cassetari, A.S.; Lima, A.S.; Brandt, E.; Pinnock, E.; Vandammec, P.; Moreira, F.M.S. 2012. Diazotrophic Burkholderia species isolated from the Amazon region exhibit phenotypical, functional and genetic diversity. Systematic and Applied Microbiology 35: 253-262.
Soares, B.L.; Ademar, P.A.; Oliveira-Longatti, S.M.; Marra, L.M.; Rufini, M. 2014. Cowpea symbiotic efficiency, pH and aluminum tolerance in nitrogen-fixing bacteria. Scientia Agricola 71: 171-180.

Soares, B.L.; Ferreira, P.A.A.; Rufini, M.; Martins, F.A.D.; Oliveira, D.P.; Reis, R.P.; Andrade, M.J.B.; Moreira, F.M.S. 2016. Agronomic and economic efficiency of commonbean inoculation with rhizobia and mineral nitrogen fertilization. Revista Brasileira de Ciência do Solo 40: 1-13.

Tan, Z.Y.; Kan, F.L.; Peng, G.X.; Wang, E.T.; Reinhald-Hurek, B.; Chen, W.X. 2001. Rhizobium yanglingense sp. nov. isolated from arid and semiarid regions in China. International Journal of Systematic Evolutionary Microbiology 51: 901-914.

Tilak, K.V.B.; Ranganayaki, R.N.; Manoharachari, C. 2006. Synergistic effects of plant-growth promoting rhizobacteria and Rhizobium on nodulation and nitrogen fixation by pigeon pea. European Journal of Soil Science 57: 67-71.

Toledo, I.; Lloret, L.; Martínez-Romero, E. 2003. Sinorhizobium americanum sp. nov., a new Sinorhizobium species nodulating native Acacia spp. in Mexico. Systematic Applied Microbiology 26: 54- 64.

Tsai, S.M.; Bonetti, R.; Agbala, S.M.; Rossetto, R. 1993. Minimizing the effect of mineral nitrogen on biological nitrogen fixation in common bean by increasing nutrient levels. Plant and Soil 152: 131-138.

Vincent, J.M. 1970. Manual for the Practical Study of Root Nodule Bacteria. Blackwell, Oxford, England.

Wang, E.T.; Rogel, M.A.; García-de los Santos, A.; MartínezRomero, J.; Cevallos, M.A.; Martínez-Romero, E. 1999. Rhizobium etli bv. mimosae, a novel biovar isolated from Mimosa affinis. International Journal of Systematic Bacteriology 49: 1479-1491. 